\title{
Linear scaling computation of the Fock matrix. VIII. Periodic boundaries for exact exchange at the $\Gamma$-point. ${ }^{1}$
}

\author{
C. J. Tymczak ${ }^{2}$ \\ Theoretical Division, Los Alamos National Laboratory, Los Alamos, New Mexico 87545 \\ Valéry T. Weber \\ Dept. of Chemistry, University of Fribourg, 1700 Fribourg, Switzerland \\ Eric Schwegler \\ Lawrence Livermore National Laboratory, Livermore, California 94551 \\ Matt Challacombe \\ Theoretical Division, Los Alamos National Laboratory, Los Alamos, New Mexico 87545
}

(Dated: April 11, 2018)

\begin{abstract}
A translationally invariant formulation of the Hartree-Fock (HF) $\Gamma$-point approximation is presented. This formulation is achieved through introduction of the Minimum Image Convention (MIC) at the level of primitive two-electron integrals, and implemented in a periodic version of the ONX algorithm [J. Chem. Phys, 1069708 (1997)] for linear scaling computation of the exchange matrix. Convergence of the HF-MIC $\Gamma$-point model to the HF $\mathbf{k}$-space limit is demonstrated for fully periodic magnesium oxide, ice and diamond. Computation of the diamond lattice constant using the HF-MIC model together with the hybrid PBE0 density functional [Theochem, 493145 (1999)] yields $a_{0}=3.569 \AA$ with the $6-21 \mathrm{G}^{*}$ basis set and a $3 \times 3 \times 3$ supercell. Linear scaling computation of the HF-MIC exchange matrix is demonstrated for diamond and ice in the condensed phase.

Keywords: Self-consistent-field, linear-scaling, periodic systems, exact-exchange, minimum image convention

PACS numbers: 71.15.-m,71.15.Ap,71.15.Mb,71.70.Gm, 31.15.Ne
\end{abstract}

\section{INTRODUCTION}

In a preceding article $\frac{1}{1}$, methods were introduced for constructing the periodic $\Gamma$-point Coulomb and Exchange-Correlation matrix that achieve a cost scaling only linearly with system size, $N$. In this paper, an $\mathcal{O}(N)$ algorithm is presented for computation of the periodic Hartree-Fock (HF) exchange matrix at the $\Gamma$-point. The Hartree-Fock approximation is often a fast, first approximation and also a starting point for correlated "wavefunction" methods. Also, hybrid Hartree-Fock/Density Functional Theory (HF/DFT) model chemistries are an important next step in accuracy beyond the Generalized Gradient Approximation 2.3.4.5. Together with linear scaling methods for computing the density matrix ${ }^{6.7}$, these advances provide a framework for the application of both $\mathrm{HF}$ and HF/DFT models to large condensed phase systems, surfaces and wires.

To date, condensed phase HF and HF/DFT calculations have been carried out almost exclusively with the $a b$ initio solid state program CRYSTAL ${ }^{8}$, which employs conventional algorithms for evaluation of the HF exchange matrix. The CRYSTAL program employs methods for $\mathbf{k}$-space integration, which generates wavefunc-

\footnotetext{
1 Preprint LA-UR 03-9043

2 tymczak@lanl.gov
}

tions with the correct translational symmetries. While the $\Gamma$-point approximation forgoes $\mathbf{k}$-space integration, it should recover the correct symmetries in the limit of a large super cell. With a more tractable formulation and asymptotic correctness in the limit of large systems, the $\Gamma$-point approximation would seem an ideal basis for linear scaling exchange algorithms. However in preliminary studies, we found that the naive HF $\Gamma$-point approximation converges to position dependent values, and that even in the large super cell limit, values different from the $\mathbf{k}$-space integration limit are approached.

In this paper we develop a translationally invariant definition of $\Gamma$-point Hartree-Fock exchange, which correctly approaches the $\mathbf{k}$-space integration value in the limit of a large super-cell. This is accomplished through introducing a Minimum Image Convention (MIC) into the exchange kernel at the level of primitive two-electron integrals, as described in Section IIII Incorporation of the MIC integrals into a periodic version of the ONX algorithm ${ }^{9}$ for linear scaling computation of the HF exchange matrix is then described in Section $\mathrm{V}$ In Section VI] we demonstrate convergence of the HF-MIC model chemistry to the $\mathbf{k}$-space integration limit for a number of systems, and in Section [II]we demonstrate linear scaling for both diamond and ice. In Section VIII we discuss these results, and then in Section $\llbracket \mathbb{I X}$ we summarize our results. 


\section{PERIODIC EXACT EXCHANGE}

In the conventional formulation of periodic boundary conditions, the Bloch functions

$$
\psi_{a}^{\mathbf{k}}(\mathbf{r})=\sum_{\mathbf{R}} e^{i \mathbf{k} \cdot \mathbf{R}} \phi_{a}(\mathbf{r}-\mathbf{R}),
$$

are often constructed from non-orthogonal functions local to the unit cell. Here, the local function $\phi_{a}$ is a GaussianType Atomic Orbital (GTAO) centered on atom A, while the sum on $\mathbf{R}$ runs over the Bravais lattice vectors defined by integer translates of the lattice primitives $\mathbf{a}, \mathbf{b}$ and $\mathbf{c}$. Inversely, the vector $\mathbf{k}$ runs over the reciprocal lattice vectors.

To date, rapid computation of the Hartree-Fock exchange interaction demands the analytic evaluation of two-electron integrals, which is possible when the local basis functions are of Cartesian Gaussian type. Typically, these functions have the form

$$
\phi_{a}(\mathbf{r})=\left(x-A_{x}\right)^{l_{a}}\left(y-A_{y}\right)^{m_{a}}\left(z-A_{z}\right)^{n_{a}} e^{-\zeta_{a}(\mathbf{r}-\mathbf{A})^{2}}
$$

where the triad $\left\{l_{a}, m_{a}, n_{a}\right\}$ sets angular symmetry and the exponent $\zeta_{a}$ is chosen to describe a particular length scale. Gaussian basis functions are often contracted to approximate atomic eigenfunctions.

With periodic boundary conditions, the exact HartreeFock exchange matrix is 8.10

$$
\mathbf{K}[\mathbf{k}]=\sum_{\mathbf{L}} \mathbf{K}[\mathbf{L}] e^{i \mathbf{k} \cdot \mathbf{L}},
$$

where

$$
K_{a b}[\mathbf{L}]=-\frac{1}{2} \sum_{\mathbf{H N}, c d} P_{c d}[\mathbf{N}]\left(\phi_{a} \phi_{c}^{\mathbf{H}} \mid \phi_{b}^{\mathbf{L}} \phi_{d}^{\mathbf{H}+\mathbf{N}}\right)
$$

is defined in real space, and the two-electron integrals, written in chemist's notation, are

$$
\begin{aligned}
& \left(\phi_{a} \phi_{c}^{\mathbf{H}} \mid \phi_{b}^{\mathbf{L}} \phi_{d}^{\mathbf{H}+\mathbf{N}}\right)= \\
& \quad \iint d \mathbf{r} d \mathbf{r}^{\prime} \frac{\phi_{a}(\mathbf{r}) \phi_{c}(\mathbf{r}+\mathbf{H}) \phi_{b}\left(\mathbf{r}^{\prime}+\mathbf{L}\right) \phi_{d}\left(\mathbf{r}^{\prime}+\mathbf{H}+\mathbf{N}\right)}{\left|\mathbf{r}-\mathbf{r}^{\prime}\right|} .
\end{aligned}
$$

The formally infinite sums over lattice vectors in Eq. (4) involve many contributions that are in practice infinitesimal. In part this is due to the decay between local basis function products $\phi_{a} \phi_{c}$; the product of two Guassians centered at $\mathbf{A}$ and $\mathbf{C}$ decays also as a Gaussian with separation $|\mathbf{A}-\mathbf{C}|$. Truncation based purely on the overlap of Gaussian basis functions is reliable and well controlled, leading to $\mathcal{O}(N)$ product terms. Additionally, the density matrix is known to decay exponentially for non-metallic systems. A cutoff defining the range of allowed exchange interactions may be used to exploit this fall off a priori by confining summation over $\mathbf{N}^{8,10,11,12,13}$ to only those terms that satisfy the imposed geometric constraints. Methods equivalent to a cutoff have also been used to achieve $N$-scaling of the exchange matrix in gas phase calculations ${ }^{14,15}$.

\section{EXCHANGE AT THE Г-POINT}

The $\Gamma$-point approximation limits $\mathbf{k}$-space sampling to just the central cell at $\mathbf{k}=0$. In the naive $\Gamma$-point limit, we take $\mathbf{P}[\mathbf{N}] \equiv \delta_{\mathbf{N}, \mathbf{0}} \mathbf{P}$, reducing Eq. (4) to the less cumbersome relation:

$$
K_{a b}=-\frac{1}{2} \sum_{\mathbf{H L}, c d} P_{c d}\left(\phi_{a} \phi_{c}^{\mathbf{H}} \mid \phi_{b} \phi_{d}^{\mathbf{L}}\right),
$$

where we have relabeled the lattice sums for clarity. However, there are two significant problems with this naive approach. First, the exact exchange potential, which is periodic in Eq. 4 has been truncated asymmetrically to yield Eq. 6] Neighboring cells correctly representing the exchange interaction have been removed, leading to a non-periodic exchange potential that varies with position of the coordinates in relation to the unit cell. Effectively, a position dependent environment has been created for each charge distribution. Secondly, this truncated expression violates symmetry of the generalized exchange energy:

$$
E_{x}\left[\mathbf{P}^{a}, \mathbf{P}^{b}\right] \neq E_{x}\left[\mathbf{P}^{b}, \mathbf{P}^{a}\right],
$$

where

$$
E_{x}\left[\mathbf{P}^{a}, \mathbf{P}^{b}\right]=\operatorname{Tr}\left[\mathbf{P}^{a} \cdot \mathbf{K}\left(\mathbf{P}^{b}\right)\right],
$$

and the superscripts label different density matrices.

Let us consider the first problem, which is the most serious defect of Eq. 6] This translational variance has a well understood analogue in classical molecular dynamics 16.17 .18 , where the arbitrary truncation of short range potentials also leads to numerical artifacts in energies and forces. In both cases, translational invariance is restored by introducing the Minimum Image Convention (MIC), which ensures that interactions are always calculated between nearest images. In computation of the Hartree-Fock exchange matrix, the MIC $\Gamma$-point approximation is just

$$
K_{a b}=-\frac{1}{2} \sum_{\mathbf{H L}, c d} P_{c d}\left(\phi_{a} \phi_{c}^{\mathbf{H}} \mid \phi_{b} \phi_{d}^{\mathbf{L}}\right)_{\mathrm{MIC}},
$$

where the MIC condition is applied in computation of the two-electron integrals at the contraction phase, ensuring that primitive charge distributions interact consistently over a minimum distance. In particular, if the primitive basis function product $\phi_{a} \phi_{c}^{\mathbf{H}}$ is centered at $\mathbf{P}$ and the primitive product $\phi_{b} \phi_{d}^{\mathbf{L}}$ is at $\mathbf{Q}$, then the minimum image convention is applied to the interaction vector $\mathbf{P Q} \equiv$ $\mathbf{P}-\mathbf{Q}$ using

$$
\begin{aligned}
\mathbf{p q} & =\mathbf{M}^{-1} \cdot \mathbf{P Q} \\
p q_{i} & =p q_{i}-\operatorname{ANINT}\left(p q_{i}-\operatorname{SIGN}\left(\delta, p q_{i}\right)\right) \\
\mathbf{P Q}_{\mathrm{MIC}} & =\mathbf{M} \cdot \mathbf{p q}
\end{aligned}
$$

where $\delta \approx 10^{-15}$ is needed to avoid wrapping errors ${ }^{39}$, and $\mathbf{M}$ is the $3 \times 3$ shape matrix of the unit cell, composed 
of the primitive lattice vectors,

$$
\mathbf{M}=\left(\begin{array}{lll}
a_{x} & b_{x} & c_{x} \\
a_{y} & b_{y} & c_{y} \\
a_{z} & b_{z} & c_{z}
\end{array}\right)
$$

This approach is completely general, and can be used at the primitive level with any modern approach to computing two-electron integrals. While the MIC yields translational invariance of the exchange matrix, it does not recover Exchange Kernel Permutational Symmetry (EKPS). With greater expense, we could recover the EKPS by explicitly symmetrizing the primitive Gaussian products within the kernel. However, in the limit of large systems, where the range of the density matrix becomes smaller than the system size, the EKPS is recovered as demonstrated in Section VI (note that, without the MIC, EKPS is not recovered in this limit).

\section{OPTIMAL DAMPING AND SYMMETRY OF THE EXCHANGE KERNEL}

For difficult, unstable SCF problems, the Optimal Damping Algorithm (ODA) of Cancés 19 is an efficient method that guarantees convergence of the HF model. However, permutational symmetry of the exchange kernel is an implicit, simplifying assumption in formulation of the conventional ODA algorithm. For small periodic systems, violation of the EKPS creates problems for the ODA, leading to a non-quadratic behavior (the HF model should yield an exactly quadratic, convex minimization problem). While the EKPS is restored with increasing system size, loose numerical thresholds can also lead to loss of the EKPS in the limit of a large system. In both cases, loss of EKPS can lead to incorrect determination of the ODA mixing parameter

$$
\lambda=\frac{\frac{d E^{0}}{d \lambda}}{3 E^{1}-3 E^{0}-2 \frac{d E^{0}}{d \lambda}-\frac{d E^{1}}{d \lambda}},
$$

where the superscripts indicate consecutive steps in the SCF cycle given by the endpoints, with $\lambda \in[0,1]$. It is of course a simple matter to reformulate the ODA, using definitions for the endpoint derivatives that do not assume EKPS:

$$
\begin{aligned}
\frac{d E^{0}}{d \lambda} & =E_{\mathrm{ne}}^{1}-E_{\mathrm{ne}}^{0}+\operatorname{Tr}\left[\left(\mathbf{P}^{1}-\mathbf{P}^{0}\right) \mathbf{T}\right] \\
& +\operatorname{Tr}\left[\mathbf{P}^{1} \mathbf{F}^{0}\right]+\operatorname{Tr}\left[\mathbf{P}^{0} \mathbf{F}^{1}\right]-2 \operatorname{Tr}\left[\mathbf{P}^{0} \mathbf{F}^{0}\right] \\
\frac{d E^{1}}{d \lambda} & =E_{\mathrm{ne}}^{1}-E_{\mathrm{ne}}^{0}+\operatorname{Tr}\left[\left(\mathbf{P}^{1}-\mathbf{P}^{0}\right) \mathbf{T}\right] \\
& -\operatorname{Tr}\left[\mathbf{P}^{1} \mathbf{F}^{0}\right]-\operatorname{Tr}\left[\mathbf{P}^{0} \mathbf{F}^{1}\right]+2 \operatorname{Tr}\left[\mathbf{P}^{1} \mathbf{F}^{1}\right]
\end{aligned}
$$

where $\mathbf{F}$ is the Fockian, $\mathbf{T}$ is the kinetic energy matrix and $E_{\text {ne }}$ is the nuclear-electrostatic energy. This modified ODA leads to the correct quadratic parameterization and guarantees convergence of the HF-MIC model.

\section{IMPLEMENTATION}

A general treatment of $\Gamma$-point periodic boundary conditions has been implemented in the MondoSCF ${ }^{20}$ suite of programs for linear scaling quantum chemistry. A detailed account of these developments for pure Density Functional Theory has been given in a companion paper ${ }^{1}$, including the periodic development of the Quantum Chemical Tree Code (QCTC) for $N$-scaling Coulomb summation. In addition to QCTC, the linear scaling, Quartic Trace-ReSetting (TRS4) ${ }^{7}$ density matrix solver has been used throughout, together with inverse congruence transformations provided by sparse atom-blocked approximate $\mathrm{AINV}^{21}$.

The Order N eXchange (ONX) algorithm ${ }^{9}$ for computing the gas phase exchange matrix has been modified by placing dual loops running over the lattice vectors $\mathbf{H}$ and $\mathbf{L}$ around the original ONX loop structures. Two ordered bra and ket distribution buffers are assembled for each lattice vector pair, which are then used to drive the basic ONX algorithm. The Minimum Image Convention has been introduced into the primitive contraction stage, in the Vertical Recurrence Relations component of a symmetry driven Head-Gordon Pople ${ }^{22}$ scheme for computing two-electron integrals.

All developments were implemented in MondoSCF v1.0 $\alpha 9^{20}$, a suite of linear scaling Quantum Chemistry codes. The code was compiled using the Portland Group F90 compiler PGF90 v4.2 $2^{23}$ with the -01 -tp athlon options and with the GNU C compiler GCC v3.2.2 using the -01 flag. All calculations were carried out on a $1.6 \mathrm{GHz}$ AMD Athlon running RedHat Linuxv9.024.

TABLE I: Progression of Hartree-Fock $\Gamma$-point super-cell calculations for $\mathrm{MgO}$ using the periodic RHF-MIC and RHF 8-511G/8-51G level of theory. Comparison is made to a final value approaching the $\mathbf{k}$-space integration limit for the primitive cell.

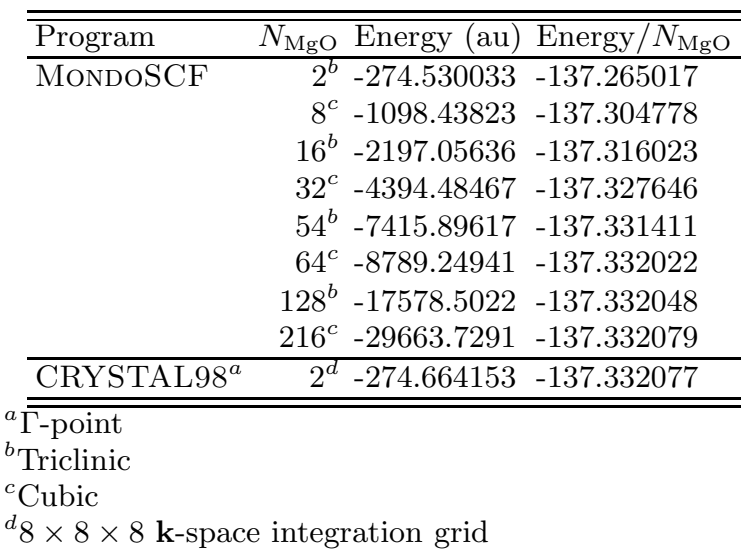


TABLE II: Progression of $\Gamma$-point super-cell calculations of proton ordered ice at the RHF-MIC/8-51G/5-11G* level of theory. Comparison is made to a final value approaching the $\mathrm{k}$-space integration limit for the primitive cell.

\begin{tabular}{lrccc}
\hline \hline Program & $N_{\mathrm{H}_{2} \mathrm{O}}$ & Energy (au) & Energy $/ N_{\mathrm{H}_{2} \mathrm{O}}$ & DEKPS \\
\hline MONDOSCF $^{a}$ & 2 & -152.03025 & -76.01512 & $10^{-3}$ \\
& 16 & -1216.3003 & -76.01877 & $10^{-4}$ \\
& 54 & -4105.0163 & -76.01882 & $10^{-5}$ \\
& 128 & -9730.4090 & -76.01882 & $10^{-6}$ \\
& 250 & -19004.705 & -76.01882 & $10^{-6}$ \\
\hline CRYSTAL98 $^{b}$ & 2 & -152.03765 & -76.01882 & \\
\hline \hline
\end{tabular}

${ }^{a} \overline{\Gamma-p o i n t}$

${ }^{b} 6 \times 6 \times 6 \mathbf{k}$-space integration grid

TABLE III: Lattice constants in $\AA$ for diamond computed using different system sizes, theory levels, and basis sets at a LOOSE accuracy. For comparison, the experimental value for diamond, extrapolated to $T=0 \mathrm{~K}$, is $\mathrm{a}_{0}=3.567 \AA$, while $\mathrm{a}_{0}=3.583 \AA$ is obtained with both the RPBE/6-31G* GGA and the RTPSS/6-31G* meta-GGA level of theory ${ }^{25}$.

\begin{tabular}{crccc}
\hline \hline Program & $N_{\text {at }}$ & Basis & $\mathrm{a}_{0}^{\mathrm{HF}}$ & $\mathrm{a}_{0}^{\text {PBE0 }}$ \\
\hline MONDOSCF $^{a}$ & 64 & STO-3G & 3.587 & 3.601 \\
- & 216 & STO-3G & 3.582 & 3.594 \\
CRYSTAL $^{b}$ & 2 & STO-3G & 3.581 & - \\
MONDOSCF $^{a}$ & 64 & $6-21 G^{*}$ & 3.575 & 3.571 \\
- & 216 & $6-21 G^{*}$ & 3.571 & 3.569 \\
CRYSTAL $^{b}$ & 2 & $6-21 G^{*}$ & 3.574 & - \\
\hline \hline
\end{tabular}

${ }^{a} \overline{\Gamma \text {-point }}$

${ }^{b}$ Taken from Ref. [26]

\section{VALIDATION}

Comparison is made to the Gaussian orbital, periodic ab initio program CRYSTAL98 ${ }^{28}$, primarily using basis sets optimized for the condensed phase, obtained from Ref. [29].

Table 【 shows the progression of total energies computed with MondoSCF for $\mathrm{MgO}$ with the $\Gamma$-point RHFMIC model, using the $8-511 \mathrm{G}$ basis set for magnesium and the 8-51G basis set for oxygen. Comparison is made to a final value approaching the $\mathbf{k}$-space integration limit for the primitive cell obtained using CRYSTAL98. The CRYSTAL basis sets were obtained from Ref. 29], and the primitive cubic and triclinic cell coordinates used for this system are given in Ref. [30]. The values controlling accuracy of the CRYSTAL98 program were obtained from Ref. [31], while the MondoSCF calculations were carried out using the TIGHT level of accuracy, defining numerical thresholds delivering numbers precise to the digits quoted.

In Table III total energies computed with the RHFMIC $\Gamma$-point super cell approach are listed for proton ordered ice $\frac{27}{2}$, using the $8-51 \mathrm{G}$ basis for oxygen and the $5-11 \mathrm{G}^{*}$ basis for hydrogen. A comparison is made to a
FIG. 1: The relaxed, uniaxial lattice potential of proton ordered ice ${ }^{27}$. Comparison is made between a RHF-MIC/851G/5-11G* 250 molecule $\Gamma$-point super-cell calculation and a CRYSTAL98 calculation carried out with a two molecule primitive cell using a $6 \times 6 \times 6 \mathrm{k}$-space integration grid.

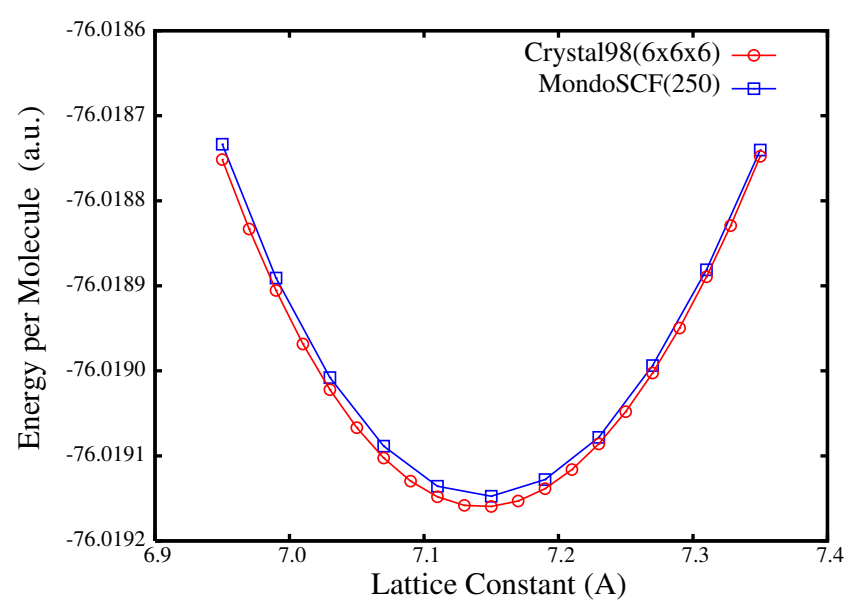

final value obtained using CyRSTAL98 with a $6 \times 6 \times 6$ $\mathbf{k}$-space integration grid as explained in Ref. 27]. The MondoSCF values were obtained using the GOOD accuracy level, defining numerical thresholds that deliver total energies precise to the digits quoted. The primitive cubic cell coordinates used in these ice calculations are listed in Ref. [30]. Also shown in this table is a measure of the Deviation from the Exchange Kernel Permutational Symmetry (DEKPS),

$$
\text { DEKPS }=\left\|\mathbf{P}^{0} \mathbf{K}^{1}-\mathbf{P}^{1} \mathbf{K}^{0}\right\|_{2} /\left\|\mathbf{P}^{1}-\mathbf{P}^{0}\right\|_{2},
$$

where the superscripts refer simply to consecutive steps in the SCF cycle. Because this measure is normalized, it yields a roughly consistent measure throughout the SCF. As shown in Table III the DEKPS decreases with system size to a constant value consistent with the numerical threshold used to discard matrix elements.

Table 11 compares the diamond lattice constant computed by MondoSCF using the RHF-MIC $\Gamma$-point supercell approximation to results obtained in reference [26. The result achieved using the 216 atom $3 \times 3 \times 3$ supercell, the $6-21 \mathrm{G}^{*}$ basis set and the hybrid PBE0 functional yields $a_{0}=3.569 \AA$ and should be compared to the experimental, $T=0 \mathrm{~K}$ result of $a_{0}=3.567 \AA$, as well as the value $a_{0}=3.583 \AA$ computed with the TPSS metaGGA $^{25}$.

Figure \shows the unrelaxed, uniaxial lattice potential of proton ordered ice 27 in the $a$ direction (see Ref. 30. for the coordinate system). Comparison is made between a 250 molecule RHF-MIC $\Gamma$-point super-cell calculation performed with MoNDOSCF and CRYSTAL98 calculations carried out with a two molecule primitive cell using a $6 \times 6 \times 6 \mathrm{k}$-space integration grid, the $8-51 \mathrm{G}$ 
basis for oxygen and the $5-11 \mathrm{G}^{*}$ basis for hydrogen. The MONDOSCF GOOD option was used, delivering a relative accuracy of 6-7 digits. The potential minimum for the CRYSTAL98 curve is $7.144 \AA$ and for the MondoSCF calculation $7.145 \AA$.

FIG. 2: CPU time for the exchange, Coulomb and density matrix build (scaled by 10) of diamond using LOOSE thresholding at the RHF-MIC/STO-3G level of theory.

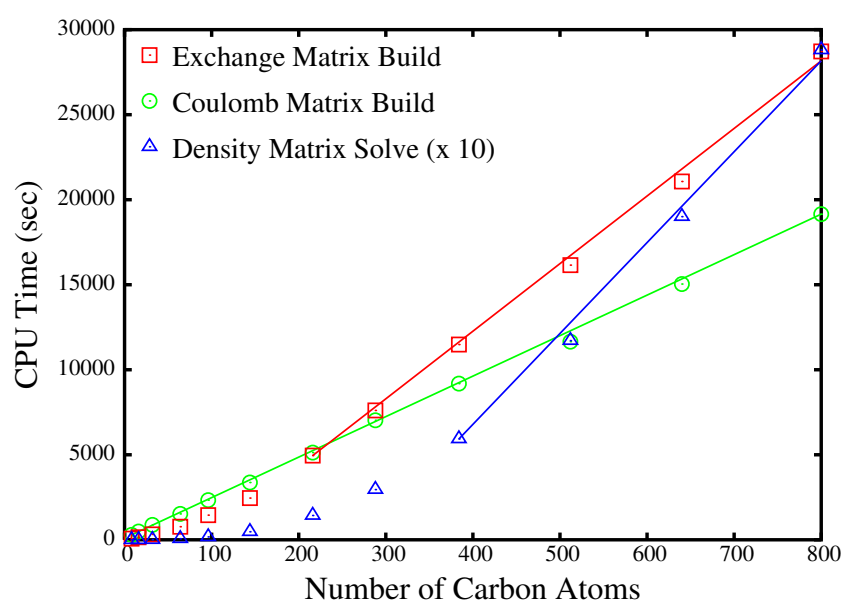

FIG. 3: CPU time for the exchange, Coulomb and density matrix build (scaled by 10) of proton ordered ice using GOOD thresholding at the RHF-MIC/8-51G/5-11G* level of theory.

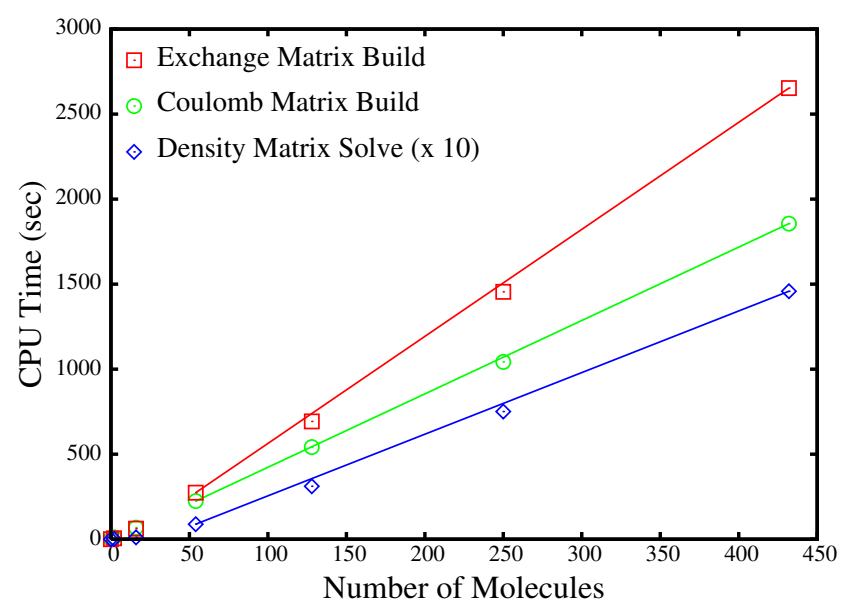

VII. SCALING

In Fig. 2 timings are shown for building the exact exchange, the Coulomb, and the density matrix of diamond at the RHF-MIC/STO-3G level of theory with a LOOSE set of thresholds. This is the same accuracy level used to compute the values listed in Table III

Figure 3 shows timings for building the exchange, Coulomb and density matrix of proton ordered ice ${ }^{27}$ at the RHF-MIC model, GOOD thresholds, the 8-51G basis set for oxygen and the $5-11 \mathrm{G}^{*}$ basis set for hydrogen. This is the level of theory used to produce Table $\amalg$ and Fig. 11 An early onset of linear scaling is observed at about 50 water molecules, which is similar to the onset achieved for water clusters in the gas phase ${ }^{7}$.

\section{DISCUSSION}

Differences between MondoSCF and CRYSTAL98 include the use of fitting functions and $\mathbf{k}$-space integration by CRYSTAL98 and the use of approximate $\mathcal{O}(N)$ scaling algorithms and the $\Gamma$-point approximation by MondoSCF. Nevertheless, in the large super cell limit, we have achieved a very close agreement between the two methods.

Importantly, the periodic MoNDOSCF algorithms have also demonstrated linear scaling for threedimensional systems. While achieving linear scaling in three-dimensions for gas phase systems is challenging, it is even more difficult with full periodicity. This is especially true for dense systems like diamond; linear scaling was achieved here only for large unit cells, a minimal basis and a LOOSE accuracy level. It is possible to achieve an early onset of linear scaling with significantly larger Gaussian basis sets by tightening the valence region, but doing so without care can lead to poor performance in the computation of proprieties such as the lattice constant. Localization of the basis set has received recent, critical attention ${ }^{32.33 .34}$, leading to numerical basis functions that yield both linear scaling and high quality properties. So far though, this issue remains unexplored in the context of a Gaussian representation.

For less dense systems and larger basis functions, the periodic MoNDOSCF algorithms are able to achieve an early onset of linear scaling with more accurate thresholding parameters. With parallelism ${ }^{35.36 .37}$, this opens the prospect of performing hybrid HF/DFT simulations of fluids.

These differences in behavior highlight the use of continuous thresholds that set the cost to accuracy ratio on the fly. Thus, as the gap closes or the basis sets becomes overcomplete, periodic ONX and TRS4 will correctly revert to $\mathcal{O}\left(N^{2}\right)$ and $\mathcal{O}\left(N^{3}\right)$ algorithms respectively. This should be compared with use of a radial cutoff to determine a priori the graph of the density and exchange matrix, which does not correctly permit fill-in.

\section{CONCLUSIONS}

For the first time, a translationally invariant definition of the periodic Hartree-Fock $\Gamma$-point approximation has been presented. Based on inserting the Minimum Image Condition (MIC) into the contraction phase of periodically summed two-electron integral algorithms, this HFMIC approach has been used to extend the ONX algo- 
rithm for linear scaling computation of the exchange matrix to periodic boundary conditions. Convergence of the HF-MIC $\Gamma$-point super-cell approximation to the $\mathbf{k}$-space integration limit has been demonstrated for $\mathrm{MgO}$ and ice to better than 8 digits. Linear scaling was demonstrated for diamond and ice, including MIC-exchange, Coulomb and density matrix construction.

\section{ACKNOWLEDGMENTS}

We would like to acknowledge Tommy Sewell and Ed Kober for their advice and support. We would also like to thank Chee Kwan Gan and Anders Niklasson for a careful reading of this manuscript.
1 C. J. Tymczak and M. Challacombe, Linear Scaling Computation of the Fock Matrix. VII. Periodic Boundary Conditions for Density Functional Theory (2004), submitted to J. Chem. Phys.

2 P. M. Gill, B. J. Johnson, J. A. Pople, and M. J. Frisch, Int. J. Quant. Chem. S26, 319 (1992).

3 A. D. Becke, J. Chem. Phys. 98, 1372 (1993).

4 V. Barone, C. Adamo, and F. Mele, Chem. Phys. Letters 249, 290 (1996).

5 C. Adamo, M. Cossi, and V. Barone, Theochem 493, 145 (1999).

6 A. M. N. Niklasson, Phys. Rev. B 66, 155115 (2002).

7 A. M. N. Niklasson, C. J. Tymczak, and M. Challacombe, J. Chem. Phys. 118(19), 8611 (2003).

8 R. Dovesi, R. Orlando, C. Roetti, C. Pisani, and V. V. R. Saunders, Phys. Stat. Sol. B 217(1), 63 (2000).

9 E. Schwegler, M. Challacombe, and M. Head-Gordon, J. Chem. Phys. 106, 9708 (1997).

10 M. Causà, R. Dovesi, C. Pisani, and V. R. Saunders, J. Phys. Chem. 92, 909 (1988).

11 R. N. Euwema, G. T. Surratt, D. L. Wilhite, and G. G. Wepfer, Bull. Amer. Phys. Soc. 19(3), 299 (1974).

12 C. Pisani and R. Dovesi, Int. J. Quant. Chem. 17(3), 501 (1980).

13 R. Dovesi, C. Pisani, and C. Roetti, Int. J. Quant. Chem. 17, 517 (1980).

14 E. Schwegler and M. Challacombe, J. Chem. Phys. 105, 2726 (1996).

15 J. C. Burant, G. E. Scuseria, and M. J. Frisch, J. Chem. Phys. 105(19), 8969 (1996).

16 N. Metropolis, A. W. Rosenbluth, M. N. Rosenbluth, A. H. Teller, and E. Teller, J. Chem. Phys. 21(6), 1087 (1953).

17 M. Allen and D. Tildesley, Computer Simulation of Liquids (Oxford Science, London, 1990).

18 M. Hloucha and U. K. Deiters, Molecular Simulation 20(4), 239 (1998).

19 E. Cances and C. L. Bris, Int. J. Quant. Chem. 79(2), 82 (2000).

20 M. Challacombe, E. Schwegler, C. J. Tymczak, C. K. Gan, K. Nemeth, V. Weber, A. Niklasson, and G. Henkelman, MondoSCF v1.0\%9, A program suite for massively parallel, linear scaling SCF theory and ab initio molecular dynamics. (2001), URL http://www.t12.lanl.gov/ mchalla/ Los Alamos National Laboratory (LA-CC 01-2), Copyright University of California.

${ }^{21}$ M. Benzi, R. K. R, and M. Tuma, Comp. Meth. App. Mech. Eng. 190(49-50), 6533 (2001).

22 M. Head-Gordon and J. A. Pople, J. Chem. Phys. 89(9), 5777 (1988).

23 The Portland Group, pgf90 v4.2 (2002), URL http://www.pgroup.com/
24 Redhat, Redhat v9.0, http://www.redhat.com/ (2004).

25 V. N. Staroverov and G. E. Scuseria, Phys. Rev. B. 69(7), $75102(2004)$

26 R. Orlando, R. Dovesi, and V. R. Saunders, J. Phys. Cond. Mat. 2(38), 7769 (1990).

27 S. Casassa, P. Ugliengo, and C. Pisani, J. Chem. Phys. 109(19), 8030 (1997).

28 V. Saunders, R. Dovesi, C. Roetti, M. Caus, N. Harrison, R. Orlando, and C. M. Zicovich-Wilson, CRYSTAL98, http://www.chimifm.unito.it/teorica/crystal/ (1998).

29 M. Towler, CRYSTAL98 Basis Sets, http://www.tcm.phy.cam.ac.uk/ mdt26/crystal.html (1998).

30 Periodic coordinates used in MondoSCF validation (2004), URL http://www.t12.lanl.gov/〜mchalla/

31 B. Civalleri, Ab Initio Modeling in Solid State Chemistry 2002, Crystal Tutorials (2002), URL http://www.crystal.unito.it/Tutorials/

32 S. D. Kenny, A. P. Horsfield, and H. Fujitani, Phys. Rev. B 62(8), 4899 (2000).

33 J. Junquera, O. Paz, D. Sanchez-Portal, and E. Artacho, Phys. Rev. B 64(23), 235111/1 (2001).

${ }^{34}$ E. Anglada, J. M. Soler, J. Junquera, and E. Artacho, Phys. Rev. B 66(20), 205101 (2002).

35 M. Challacombe, Comput. Phys. Commun. 128, 93 (2000).

36 C. K. Gan and M. Challacombe, J. Chem. Phys. 118, 9128 (2003).

37 C. K. Gan, C. J. Tymczak, and M. Challacombe, Linear Scaling Computation of the Fock Matrix. IX. Parallel Computation of the Coulomb Matrix. (2004), submitted to J. Chem. Phys.

38 M. Challacombe, J. Chem. Phys. 113, 10037 (2000).

$39 \delta$ is required to yield a consistent wrapping when distributions lie exactly at the cell boundary. In effect, this implementation changes the wrapping condition from $\left|p q_{i}\right| \geq 1$ to $\left|p q_{i}\right|>1$. ANINT $(x)$ rounds $x$ to the closest integer and $\operatorname{SIGN}(x, y)$ transfers the sign from $y$ to $x$.

\section{APPENDIX A: THRESHOLDS}

There are four thresholds that control the numerical precision of the linear scaling algorithms in MoNDOSCF. They are the matrix threshold $\tau_{\text {MTRIX }}$ described in Ref. 7], the two-electron threshold $\tau_{2 \mathrm{E}}$ (thresh in Ref. 9]), the distribution threshold $\tau_{\text {DIST }}$ described in Ref. 1], and the $\mathrm{HiCu}$ threshold $\tau_{\text {Hicu }}$ used to set the exchange-correlation grid $\left(\tau_{r}\right.$ in Ref. 38$\left.]\right)$. The threshold $\tau_{2 \mathrm{E}}$ also controls accuracy of the Coulomb matrix as discussed in Ref. [1]. These thresholds, listed in Table IV 
have been calibrated over a wide range of systems to yield a minimum of 4,6 and 8 digits of relative accuracy in the total energy with LOOSE, GOOD and TIGHT respectively. Typically however, one additional digit is achieved for models that contain Hartree-Fock exchange.

TABLE IV: Accuracy goals and corresponding thresholds that control precision of the MoNDOSCF linear scaling algorithms.

\begin{tabular}{lllll}
\hline \hline Accuracy & $\tau_{\text {MTRIX }}$ & $\tau_{2 \mathrm{E}}$ & $\tau_{\text {DIST }}$ & $\tau_{\text {HICU }}$ \\
\hline LOOSE & $10^{-4}$ & $10^{-6}$ & $10^{-8}$ & $10^{-3}$ \\
GOOD & $10^{-5}$ & $10^{-8}$ & $10^{-10}$ & $10^{-5}$ \\
TIGHT & $10^{-6}$ & $10^{-10}$ & $10^{-12}$ & $10^{-7}$ \\
\hline \hline
\end{tabular}

